\title{
HDR Pathological Image Enhancement Based on Improved Bias Field Correction and Guided Image Filter
}

\author{
Qingjiao Sun, ${ }^{1}$ Huiyan Jiang, ${ }^{1}$ Ganzheng Zhu, ${ }^{1}$ Siqi Li, ${ }^{1}$ Shang Gong, \\ Benqiang Yang, ${ }^{2}$ and Libo Zhang ${ }^{2}$ \\ ${ }^{1}$ Software College of Northeastern University, Shenyang, Liaoning 110819, China \\ ${ }^{2}$ Department of Radiology, Chinese PLA General Hospital, Shenyang 110015, China \\ Correspondence should be addressed to Huiyan Jiang; jianghy@swc.neu.edu.cn
}

Received 4 September 2016; Revised 18 November 2016; Accepted 8 December 2016

Academic Editor: Enzo Terreno

Copyright (C) 2016 Qingjiao Sun et al. This is an open access article distributed under the Creative Commons Attribution License, which permits unrestricted use, distribution, and reproduction in any medium, provided the original work is properly cited.

Pathological image enhancement is a significant topic in the field of pathological image processing. This paper proposes a high dynamic range (HDR) pathological image enhancement method based on improved bias field correction and guided image filter (GIF). Firstly, a preprocessing including stain normalization and wavelet denoising is performed for Haematoxylin and Eosin (H and E) stained pathological image. Then, an improved bias field correction model is developed to enhance the influence of light for high-frequency part in image and correct the intensity inhomogeneity and detail discontinuity of image. Next, HDR pathological image is generated based on least square method using low dynamic range (LDR) image, $\mathrm{H}$ and $\mathrm{E}$ channel images. Finally, the fine enhanced image is acquired after the detail enhancement process. Experiments with 140 pathological images demonstrate the performance advantages of our proposed method as compared with related work.

\section{Introduction}

Pathological image is an important basis for computer aided diagnosis and is regarded as the gold standard in disease diagnosis. Cell segmentation and identification are critical steps in various medical diagnoses; it is difficult to acquire accurate cell segmentation results because of low contrast and noise of image. To address this issue, it is necessary to enhance pathological image before cell segmentation process. The enhancement of pathological image can improve image quality and contrast, and it can provide more objective and reliable data support for doctors. This is of great significance and strong application value as it improves detection efficiency and medical diagnostic accuracy, meanwhile, reducing manual diagnosis error and human costs.

There are a variety of image enhancement methods and frameworks to improve image quality. Traditional histogram equalization- (HE-) based methods [1-3] are widely used for image enhancement owing to their simplicity. Besides, many new models and algorithms are proposed to process image. Reference [4] proposed an efficient transformation- free approach for color image enhancement, which manipulates pixels value directly in source RGB color space. Reference [5] presented a color image enhancement method using daubechies wavelet transform and HIS color space. Reference [6] enhanced the pathological anatomy images based on superresolution to improve medical diagnosis. In recent years, the Retinex theory is widely used in the medical image processing [7]. Many algorithms based on Retinex theory such as single-scale Retinex (SSR) [8], multiscale Retinex (MSR) [9], McCann99 [10], and Frackle-McCann [11] have been developed for image enhancement. There are also many other methods to enhance high dynamic range (HDR) images [12-14]. Reference [15] put forward a Bilateral Filtering-Dynamic Range Partitioning (BF-DRP) algorithm which can use bilateral filter (BF) on image to extract a coarse component and a details component which are processed independently and then recombined together to obtain final enhanced image. Reference [16] improved the BF-DRP algorithm by adding an adaptive Gaussian filter to smooth the imbalanced variation of gradient. Reference [17] proposed guided image filter (GIF) and [18] applied that in 
HDR image denoising and enhancement, which can avoid gradient flipping artifacts.

However, all these methods mainly target natural images or infrared images; the effect is unsatisfactory when they are used on pathological images. It remains a challenging task to obtain a good enhancement result for color pathological images because of three main issues: intensity inhomogeneity of image, detail discontinuity in tissue structures, and lower dynamic range of image. To address those issues, in this paper, we propose a HDR pathological image enhancement method based on GIF and improved bias field correction model. The main contributions of this paper are as follows. First, a new pathological image operational process is designed to denoise and enhance the source low dynamic range (LDR) image. Second, an improved bias field correction model is proposed to correct the intensity inhomogeneity and detail discontinuity of image. Third, a new method to generate HDR pathological image is presented, using LDR image and Haematoxylin $(\mathrm{H})$ and Eosin (E) channel image after stain separation.

The remainder of the paper is organized as follows. In Section 2, we introduce the related work. Our proposed pathological image enhancement method is described in Section 3. Section 4 shows our experiments results and compares them with other enhancement methods. Finally, conclusions are summarized in Section 5.

\section{Related Work}

2.1. Bias Field Correction Model. The intensity inhomogeneity is a common phenomenon of medical images, which is attributed to many factors, such as nonuniform illumination, imaging equipment defect, and the complexity of human tissues. Intensity inhomogeneity is a critical factor that affects some image processing, because it will influence the true intensity region of different tissues and then lead to the errors of image segmentation or other image analysis processes. Therefore, it is one necessary step to remove the intensity inhomogeneity from the image. The bias field is a popular mathematical assumption of image intensity inhomogeneity, which is generally accepted at present, and it manifested as the smoothly varying of intensity within the same tissue of the image. This assumption can be represented by the following mathematical model [19]:

$$
I_{o}=B \times J+n,
$$

where $I_{o}$ is the observed image with intensity inhomogeneity, $J$ is the true image, $B$ is the bias field, and $n$ is Gaussian noise with zero mean which can be ignored after denoising process and then get $I_{o}=B \times J$. There are usually two assumptions for the above model [20]:

(1) The bias field $B$ is smoothly varying. That is, the bias field approximates a constant in a small neighborhood of every pixel in the observed image.

(2) The true image $J$ describes the physical property of tissues and the value of this property should be the same in the same tissue. Thus we assume that the pixel value within every tissue of the true image $J$ is a constant.
The bias field correction procedure is used to remove the bias field from image and finally to obtain the corrected image $I_{o} / B$. There are many bias field correction methods, and [20] proposed a new algorithm called multiplicative intrinsic component optimization (MICO) for bias field estimation, which achieved a good result. In that paper, the bias field is represented by a linear combination of a group of smooth basis functions $g_{1}, \ldots, g_{M}$ as follows:

$$
B(x)=W^{T} G(x),
$$

where $G(x)=\left(g_{1}(x), \ldots, g_{M}(x)\right)^{T}$ is a column vector valued function composed of basis functions, $(\cdot)^{T}$ is the transpose operator, and $x$ is pixel point in the image, and $W=\left(\omega_{1}, \ldots, \omega_{M}\right)^{T}$ is a column vector of the coefficients. Therefore, bias field estimation can be viewed as to find the optimal coefficients $\omega_{1}, \ldots, \omega_{M}$ of the linear combination $B(x)=\sum_{k=1}^{M} \omega_{k} g_{k}$.

In addition, the true image is formulated as the following model:

$$
J(x)=\sum_{i=1}^{N} c_{i} \mu_{i}(x),
$$

where $c_{i}$ is the constant of the $i$ th tissue and there are $N$ tissues in the image, $\mu_{i}(x)$ is the percentage of the $i$ th tissue being in the pixel $x$, there are $\mu_{i}(x)=1$ for $x \in \Omega_{i}$ and $\mu_{i}(x)=0$ for $x \notin \Omega_{i}$, and $\Omega_{i}$ is the range of $i$ th tissue.

In order to calculate the bias field $B$, that paper proposed an energy minimization function as follows:

$$
F(B, J)=\int_{\Omega}\left|I_{0}(x)-B(x) J(x)\right|^{2} d x
$$

where $\Omega$ is the whole image domain. After merging equations (2), (3), and (4), the energy function $F$ can be expressed as

$$
\begin{aligned}
F(B, J) & =F(\mu, c, \omega) \\
& =\int_{\Omega}\left|I_{0}(x)-W^{T} G(x) \sum_{i=1}^{N} c_{i} \mu_{i}(x)\right|^{2} d x .
\end{aligned}
$$

We can see that the energy $F$ is the function about variables $\mu, c, \omega$. The minimization of $F$ can be achieved by alternately solving one variable with the other two fixed. And we can finally obtain the bias field corrected image after solving the bias field estimation.

2.2. Guided Image Filter. GIF filters the input image by considering the guidance image. It is a smoothing operator which can smooth filtering, preserve the edge details, and avoid the artifacts effectively. It is fast and easy to implement and can obtain a nice visual quality. GIF is derived from a local linear transformation model considering the content of a guidance image. The filtering process at a pixel $i$ can be formulated as follows [17]:

$$
q_{i}=a_{k} I_{i}+b_{k}, \quad \forall i \in \omega_{k}
$$




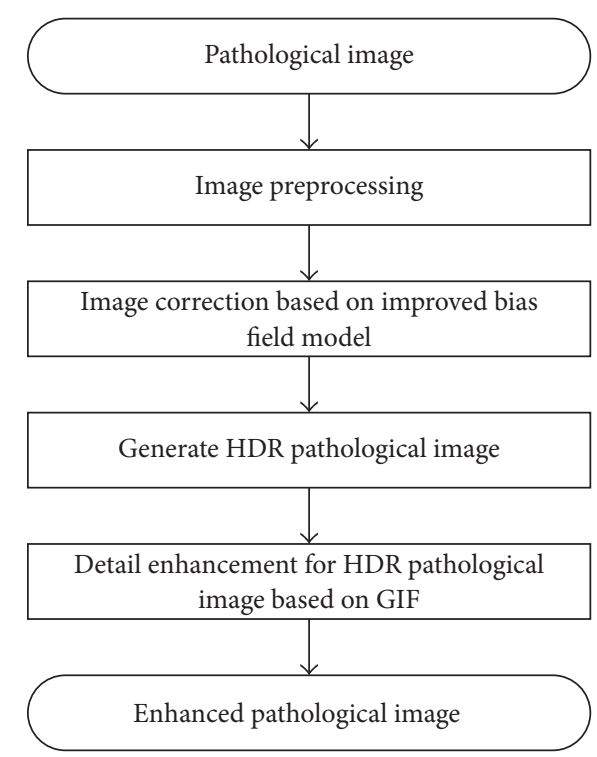

FIGURE 1: The flowchart of proposed pathological image enhancement method.

where $I$ is the guidance image and the $q$ is the linear transform of $I$ in window $\omega_{k}$ centered at pixel $k .\left(a_{k}, b_{k}\right)$ are the local linear coefficients, the calculating formula of which is as follows:

$$
\begin{aligned}
& a_{k}=\frac{(1 /|\omega|) \sum_{i \in \omega_{k}} I_{i} p_{i}-\mu_{k} \bar{p}_{k}}{\sigma_{k}^{2}+\varepsilon}, \\
& b_{k}=\bar{p}_{k}-a_{k} \mu_{k},
\end{aligned}
$$

where $\mu_{k}$ and $\sigma_{k}^{2}$ are the mean value and variance separately of image $I$ in window $\omega_{k},|\omega|$ is the number of pixels within $\omega_{k}, p$ is the input image, and $\bar{p}_{k}$ is the mean of $p$ in window $\omega_{k} \cdot \varepsilon$ is the regularization parameter also called smooth factor and used to prevent $a_{k}$ being too large.

However, a pixel $i$ is involved in more than one window that covers $i$ when computing (2) and $q_{i}$ has different value in different windows. Therefore, we can get the final $q_{i}$ value by averaging all $q_{i}$ and thus the output of the filter can be represented as follows:

$$
q_{i}=\bar{a}_{i} I_{i}+\bar{b}_{i} .
$$

Here, the average local linear coefficients are $\overline{a_{i}}=$ $(1 /|\omega|) \sum_{k \in \omega_{i}} a_{k}$ and $\bar{b}_{i}=(1 /|\omega|) \sum_{k \in \omega_{i}} b_{k}$.

\section{Proposed Pathological Image Enhancement Method}

As shown in Figure 1, the generic process of proposed pathological image enhancement method is introduced.

3.1. Image Preprocessing. There are always some undesirable problems such as hypochromasia, hyperchromasia, and color variations in the pathological images acquired from stained tissue due to different staining solutions of manufactures, different scanners or microscopes, and different staining protocols of labs, which can block the image observation and image interpretation [21]. To improve image quality, we use Reinhard's stain normalization method [22] to bring the pathological image into a better color appearance of a target image selected by pathologists.

There is much additive noise in the pathological image after stain normalization; thus we adopt denoising method to smooth the image. Some denoising methods often cannot take into account both denoising and preserving image detail and lead to edge blur problem. To solve this problem, the wavelet denoising method [23] is performed, which can preserve the image edge and other feature information while denoising.

3.2. Improved Bias Field Correction Model. The traditional bias field model concerns the intensity inhomogeneity caused by bias field in the low frequency part but ignores the detail discontinuity of high-frequency part caused by different light. Therefore, we improve the bias field model through including the detail discontinuity:

$$
I_{o}=B \times D \times J+n,
$$

where $D$ is the factor of detail discontinuity.

We have the following assumptions about factor $D$ :

(1) $D$ is mainly high-frequency information and only exists in the edge region of image.

(2) $D$ is slowly varying in the edge region and has no significant gradient variation.

After denoising, (9) becomes $I_{o}=B \times D \times J$. At the base of MICO algorithm [20], we use high-pass filter to remove low frequency information and then superimpose high-frequency information to enhance image details and to remove detail discontinuity.

3.3. Generate HDR Pathological Image. The dynamic range of pathological image acquired by digital image acquisition devices is very limited. Generally, the luminance range of digital image described by RGB color model is about two orders of magnitude (256) and there are always some overexposure and underexposure parts in the image. However, the dynamic range of a real scene is much wider than that of the image and the range that can be observed by human eyes is also much larger. In medical diagnosis, the dynamic range of sensors can reach $10^{4}$, even $10^{5}$, but LDR image cannot record all information. On the contrary, HDR image can record both RGB information and actual luminance information of pixels and can describe image details better. We could provide better images with clearer details and more reliable information for subsequent cell segmentation and identification if using LDR images to generate HDR image. It is noted that HDR images need to be compressed because they cannot be displayed on traditional screen directly, which is the tone mapping operator (TMO) of HDR image [24]. Therefore, HDR image enhancement can change the dynamic range of luminance of original image, meanwhile acquiring more details of the real scene. 
TABle 1: Comparison of GIF and BF in run time (unit: second).

\begin{tabular}{|c|c|c|c|c|c|}
\hline \multirow{2}{*}{ Methods } & \multicolumn{5}{|c|}{ Images } \\
\hline & Group $1(1280 \times 960)$ & Group $2(640 \times 480)$ & Group $3(320 \times 240)$ & Group $4(100 \times 100)$ & Group $5(50 \times 50)$ \\
\hline GIF & 1.0483 & 6.0847 & 0.0506 & 0.0285 & 0.0262 \\
\hline $\mathrm{BF}$ & 22.6512 & 0.2554 & 1.8595 & 0.5634 & 0.3692 \\
\hline
\end{tabular}

We usually need many image sequences with different exposure of the same scene to generate HDR image. But in this paper, we generate HDR image sequences based on stain separation method [25]. In this case, we cannot determine the exposure time. Here one sample strategy is that we estimate the exposure time by calculating the ratio of mean values of the sequential images.

The steps of generating HDR image are as follows:

(1) Separate the corrected pathology image into H channel stained by Haematoxylin and E channel stained by Eosin based on the stain separation method.

(2) Estimate the ratio of exposure time of adjacent images based on their means of pixels for both corrected pathology images and $\mathrm{H}$ and $\mathrm{E}$ channel images. Then fit curve of pixel relationship based on least square method.

(3) Calculate camera response function based on Mitsunaga algorithm [26] by replacing the pixel value with pixel relationship curve and then generate HDR pathological image.

3.4. Detail Enhancement Based on GIF. The enhancement method based on BF [27] can reduce the loss of image detail information, but it has gradient reverse problem. In this paper we replace BF with GIF, because GIF can avoid image gradient reverse and reduce calculation cost. In order to prove the superiority of GIF, we design five groups of images with different sizes. And there are 10 color pathological images with the same size in each group. Every image is processed with $\mathrm{BF}$ and GIF, respectively, and the run time is recorded successively. Finally we calculate the average run time for every group. The comparison result is shown in Table 1.

It is clear that GIF takes much less run time than BF when processing the same image. Therefore in our image enhancement method, we firstly transform the HDR pathology image into $\mathrm{YCbCr}$ color space (the type of sampling is $4: 4: 4$ ), then divide the luminance component into base layer and detail layer by GIF rather than BF, next compress the dynamic range for base layer using histogram mapping to reduce noise and enhance detail information, meanwhile enhance the detail layer by adaptive masking, and finally combine the enhanced luminance component and color component together. The basic procedure is shown in Figure 2.

The equation of hierarchical processing for image based on GIF is shown as follows:

$$
\begin{aligned}
& I_{B}(x, y)=F_{G}\left(I_{Y}(x, y)\right), \\
& I_{D}(x, y)=I_{Y}(x, y)-I_{B}(x, y),
\end{aligned}
$$

where $F_{G}$ is the GIF, $I_{Y}$ is the logarithm of luminance $Y$ after transforming the HDR image into $\mathrm{YCbCr}$ color space, $I_{B}$ is the base layer of image including the grey information of texture region in image, and $I_{D}$ is the detail layer including highfrequency detail information such as edges. $(x, y)$ describe the coordinate of the pixel.

The result of GIF is related to the radius $r$ of filter window and the regular parameter (smooth factor) $\varepsilon$. Figure 3 shows how these two parameters affect the hierarchical results for pathology image. Figure 3(a) is the original pathological image and (b) is the Y channel image by transforming the original image into $\mathrm{YCbCr}$ color space. (c), (e), and (g) show the base layer with $r=6, \varepsilon=0.04, r=10, \varepsilon=0.01$, and $r=10, \varepsilon=0.04$, respectively. (d), (f), and (h) show the detail layer of image corresponding to (c), (e), and (g), respectively. It is seen that the detail layer with larger window radius and regular parameter can obtain much more detail information.

\section{Experiments and Comparison}

In this paper, all the experiments are implemented using Matlab R2014a development tool in the 64-bit Windows 7 operating system (8-core CPU, 3.40 GHz, 8 G memory), and our experimental data is actual clinical pathological images which are RGB color images stained by Haematoxylin and Eosin ( $\mathrm{H}$ and $\mathrm{E})$. The picture format is TIFF and spatial resolution is $1280 * 960$. The data includes 123 liver tissue images and 17 lung tissue images, that is, 140 images in total. Besides, the parameters of the GIF are $r=10, \varepsilon=$ 0.04 . The enhancement result of our proposed method is shown in Figure 4. Figure 4(a) is the original pathological image and Figure 4(b) is the reference image which has good stain quality and is used in the stain normalization process. Figure 4(c) is the normalized image after stain normalization. Figure $4(\mathrm{~d})$ is the result after denoising and correcting by improved bias field model. Figures 4(e) and 4(f) show the $\mathrm{E}$ channel and $\mathrm{H}$ channel images after stain separation of Figure 4(d), respectively. Figure 4(g) is the HDR pathological image generated with Figures 4(c), 4(e), and 4(f), displayed by TMO. Figure $4(\mathrm{~h})$ is the final detail enhanced HDR pathological image.

It is observed that our proposed method can obtain a good image enhancement effect in Figure 4. This method can improve image luminance and contrast; meanwhile it can preserve the image detail structure well. In order to verify the effectiveness of our algorithm, we compare our method with various Retinex-based methods and some HE-based algorithms, namely, Frankle-McCann Retinex [11], SSR [28], MSR [29], Dualistic Sub-Image Histogram Equalization 


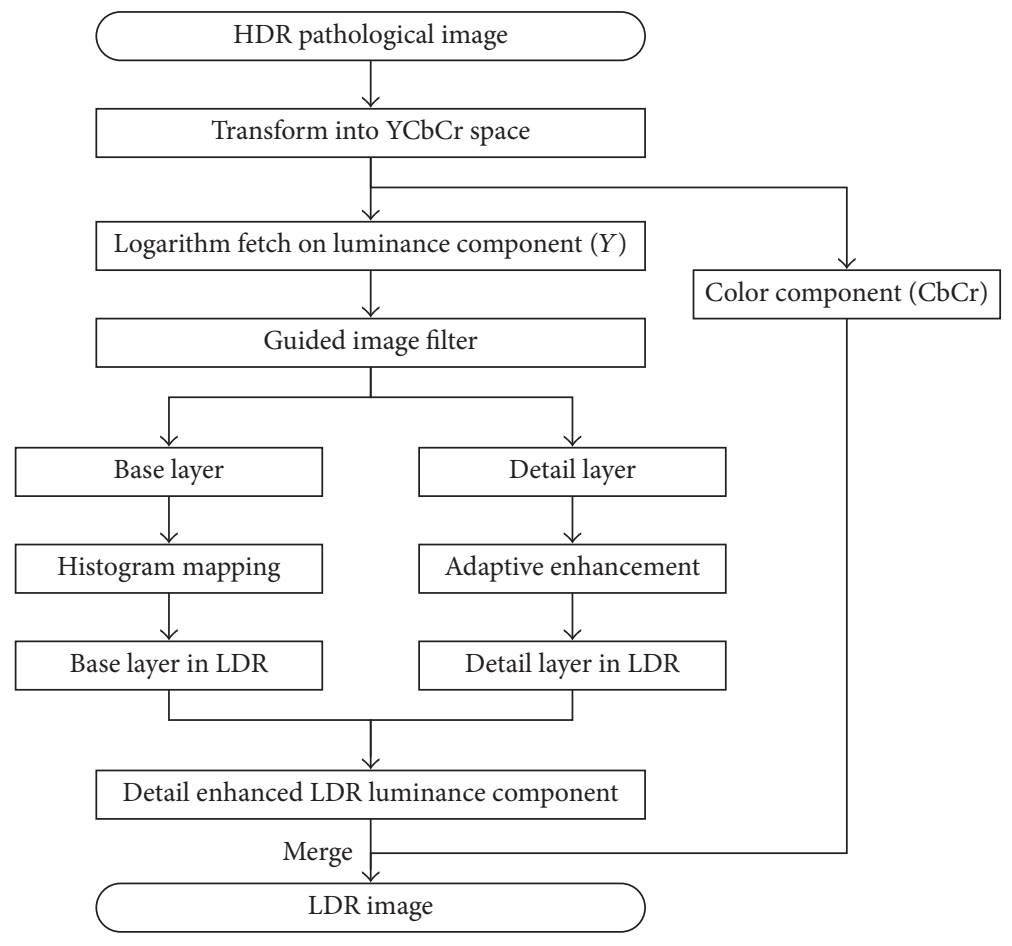

FIGURE 2: The flowchart of detail enhancement based on GIF.

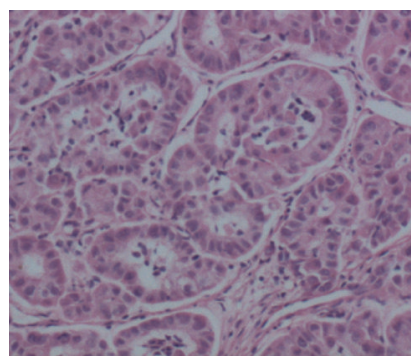

(a) Original image

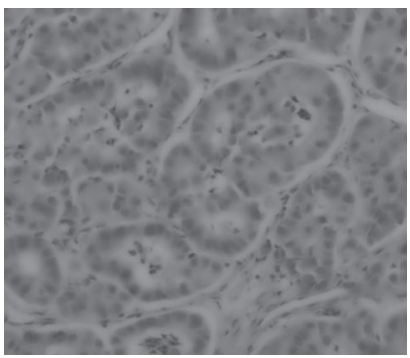

(e) Base layer image $(r=10, \varepsilon=$ 0.01 )

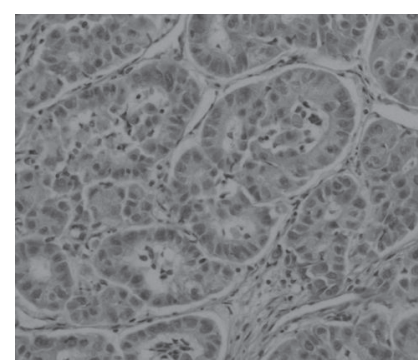

(b) Y channel image

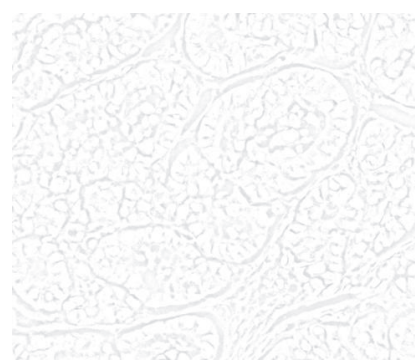

(f) Detail layer image $(r=10, \varepsilon=$ 0.01)

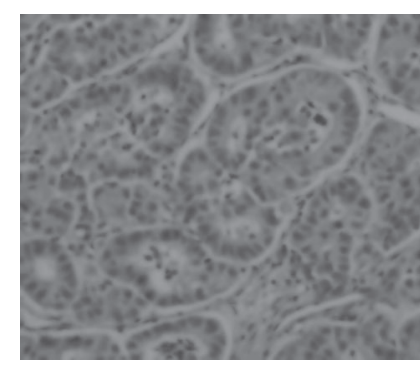

(c) Base layer image $(r=6, \varepsilon=0.04)$

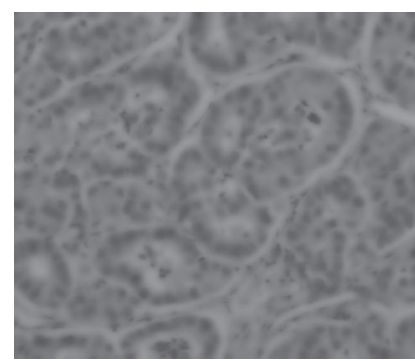

(g) Base layer image $(r=10, \varepsilon=$ 0.04)

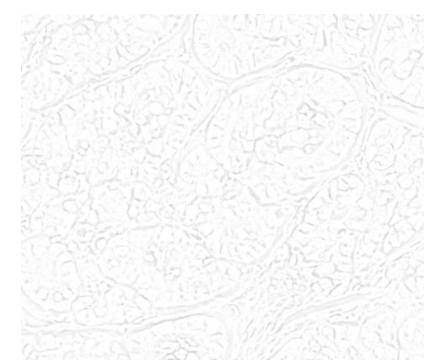

(d) Detail layer image $(r=6, \varepsilon=$ 0.04 )

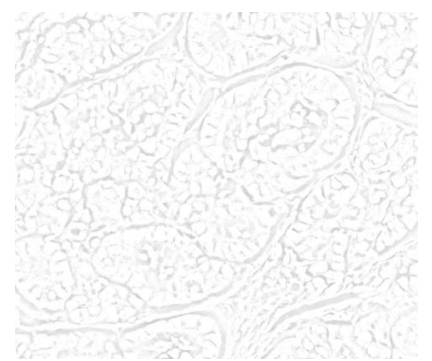

(h) Detail layer image $(r=10, \varepsilon=$ 0.04

FIGURE 3: Illustration of layer separation on pathological image with different parameters.

(DSIHE) [30], Minimum Mean Brightness Error Bi-Histogram Equalization (MMBEBHE) [31], Recursive MeanSeparate Histogram Equalization (RMSHE) [32], and Recursive Sub-Image Histogram Equalization (RSIHE) [33]. All these methods are applied to our 140 pathological images successively and we finally get 140 groups of enhanced images. There are 9 images in every group: one original image and 8 result images enhanced by 8 image enhancement algorithms. Figure 5 demonstrates one of the groups, where Figure 5(a) is the original image, Figure 5(b)-Figure 5(h) are the enhanced 


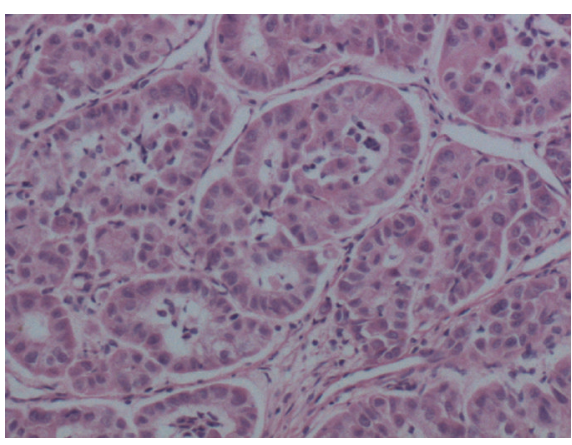

(a) Original image

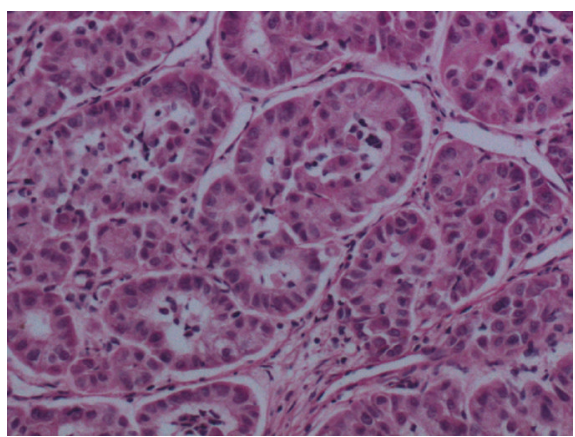

(c) Normalized image

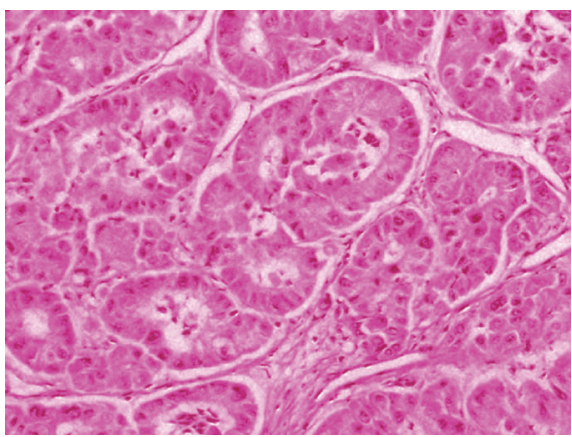

(e) E channel image

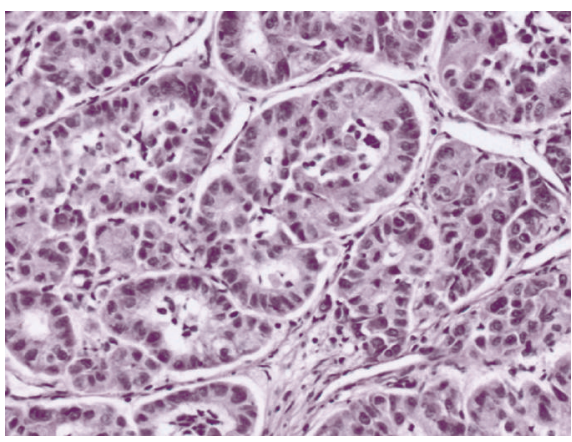

(g) HDR image after tone mapping

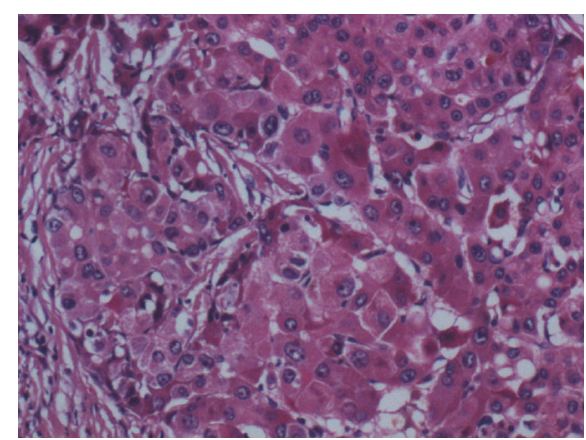

(b) Reference image for stain normalization

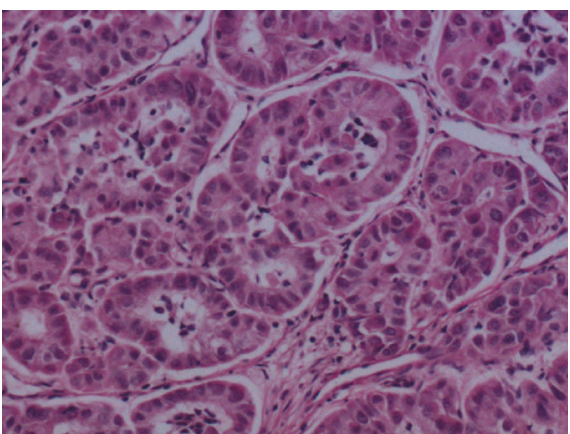

(d) Corrected image by improved bias field model

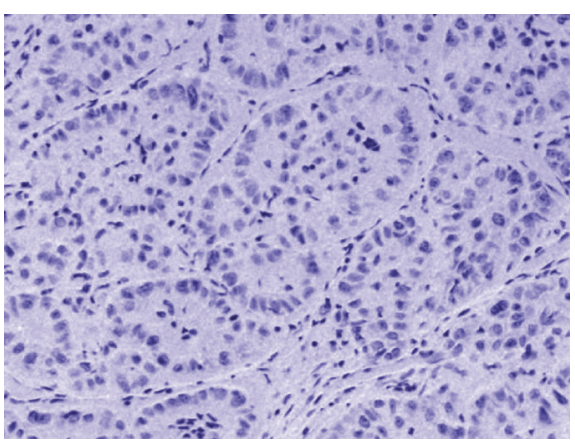

(f) $\mathrm{H}$ channel image

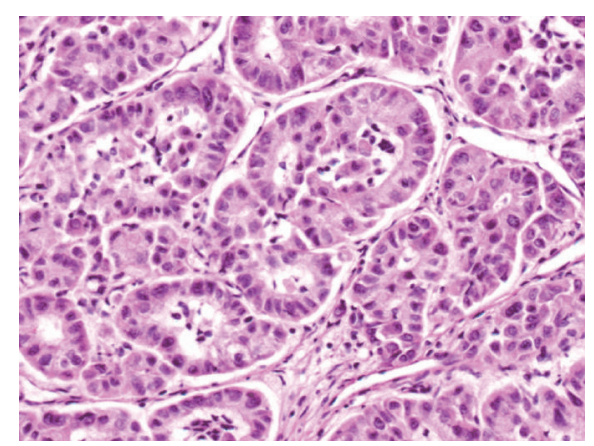

(h) Detail enhanced HDR image

FIGURE 4: The result of our proposed HDR pathological image enhancement method.

image by the above comparison algorithms, respectively, and Figure 5(i) is our result.

We can see from Figure 5 that all the above methods can enhance the pathological images to some extent. But the differences between the results are a little big. To evaluate correctly the enhancement results of different algorithms, this paper analyze the corresponding images from both the subjective and objective aspects. 


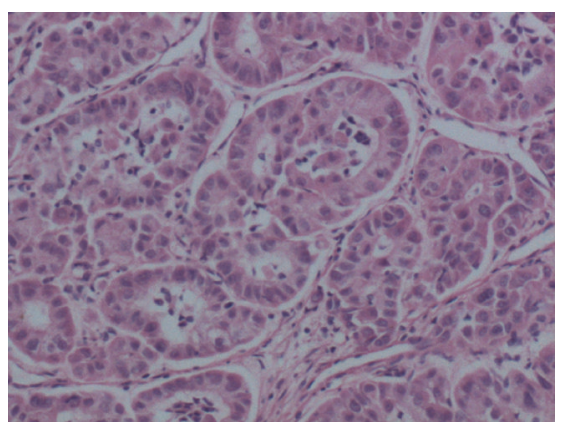

(a) Original image

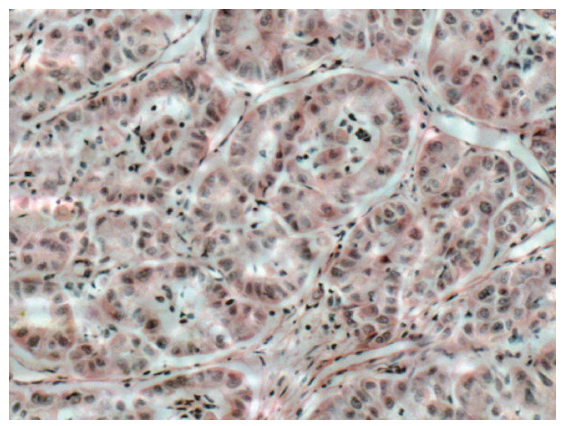

(d) MSR

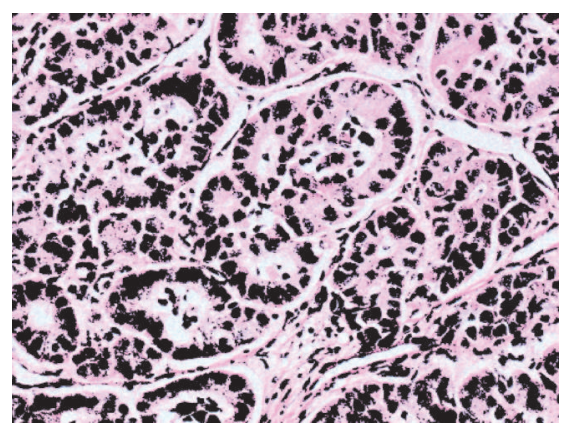

(g) RMSHE

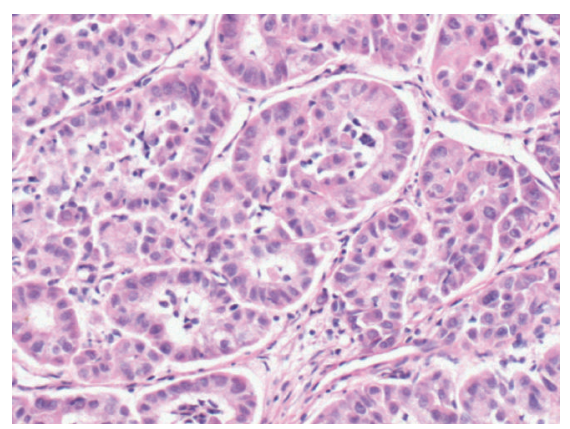

(b) Frankle-McCann Retinex

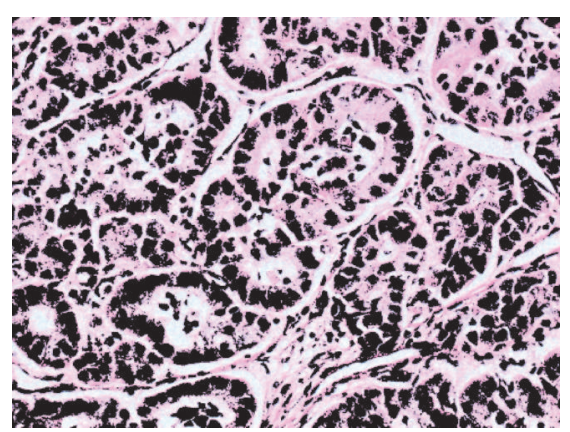

(e) DSIHE

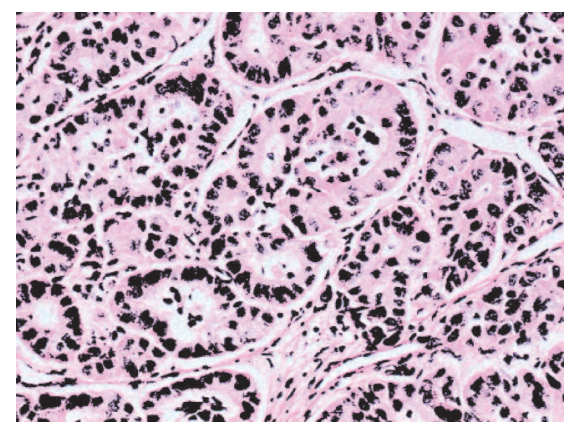

(h) RSIHE

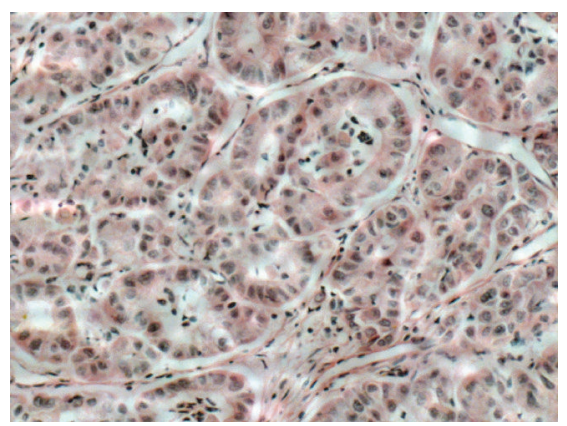

(c) SSR

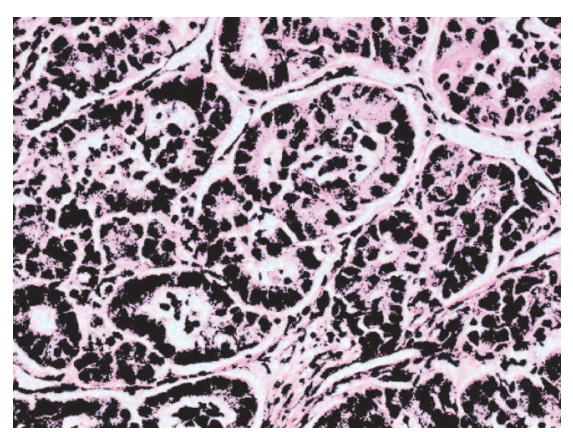

(f) MMBEBHE

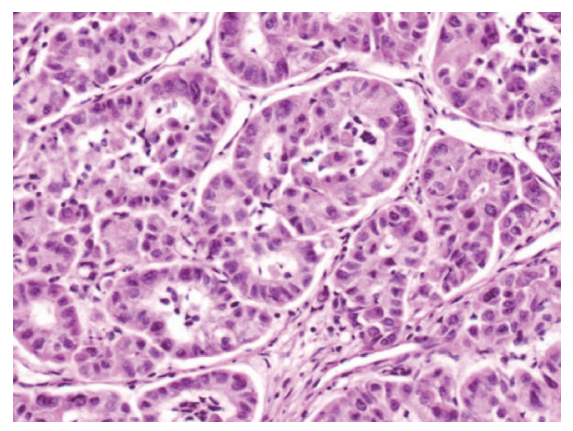

(i) Proposed

FIGURE 5: The comparison of our method with other different image enhancement methods.

TABLE 2: The number of best enhanced image selected by pathologists for different algorithms.

\begin{tabular}{lcccccccccc}
\hline & Original image & Frankle-McCann & SSR & MSR & DSIHE & MMBEBHE & RMSHE & RSIHE & Proposed \\
\hline Pathologist 1 & 0 & 4 & 11 & 13 & 0 & 0 & 0 & 0 & 22 \\
Pathologist 2 & 0 & 3 & 12 & 15 & 0 & 0 & 0 & 0 & 20 \\
Pathologist 3 & 1 & 3 & 10 & 15 & 0 & 0 & 0 & 1 & 20 \\
\hline
\end{tabular}

4.1. Subjective Evaluation. Pathological image is the gold standard in disease diagnosis. In order to verify the practicability of our algorithm, we extract 50 groups of images randomly and invite three pathologists to choose one best image from every group. The selected image should be the optimal in visual quality and the most helpful to clinical application from the perspective of a pathologist. The final result is shown in Table 2 .
As Table 2 shows, the results of HE-based algorithms are basically not recognized by pathologists, because there is serious distortion problem in the enhanced image of those algorithms. Most of the detail information in those images is lost and the cell regions cannot be distinguished. Although it is possible to obtain the optimal enhancement effect for every one of the other algorithms, our proposed method is better statistically, having the maximum amount of the selected best 
TABLE 3: Data comparison of our method with other different image enhancement methods.

\begin{tabular}{|c|c|c|c|c|c|c|}
\hline \multirow{2}{*}{ Algorithms } & \multicolumn{6}{|c|}{ Evaluations } \\
\hline & PSNR (dB) & SD & Mean & EME & Entropy (bit) & Run time (second) \\
\hline Original image & & 19.6614 & 132.1559 & 5.0345 & 6.4188 & - \\
\hline Frankle-McCann Retinex & 10.8521 & 31.1252 & 203.9961 & 5.3093 & 7.0448 & 13.6394 \\
\hline SSR & 14.5729 & 38.7156 & 171.2390 & 7.6546 & 7.2010 & 7.6829 \\
\hline MSR & 15.0145 & 38.1384 & 168.5765 & 7.7727 & 7.1866 & 21.7126 \\
\hline DSIHE & 7.9528 & 114.8437 & 150.2071 & 0.5732 & 4.4251 & 1.1934 \\
\hline MMBEBHE & 7.8207 & 117.6716 & 138.5599 & 0.4534 & 4.1568 & 0.9758 \\
\hline RMSHE & 8.0553 & 111.2631 & 160.3926 & 0.7006 & 4.6547 & 0.9987 \\
\hline RSIHE & 8.2315 & 97.6331 & 184.7367 & 1.0570 & 5.2014 & 1.1761 \\
\hline Proposed & 12.2576 & 43.9754 & 205.2157 & 8.2986 & 7.3341 & 3.7206 \\
\hline
\end{tabular}

image. So from a pathologist's viewpoint, our pathological image enhancement method can improve the image quality a lot.

4.2. Objective Evaluation. On the other hand, we also analyze the experiment results quantitatively in five well-known metrics, namely, peak signal noise ratio (PSNR) [34], standard deviation (SD), mean, measure of enhancement (EME) [35], and information entropy [34].

PSNR is widely used to evaluate the quality of image. The higher PSNR value denotes that the image could suppress noise better. The calculation equation of PSNR is as follows:

$$
\text { PSNR }=\frac{10 \log _{10}(L-1)^{2}}{\mathrm{MSE}}(\mathrm{dB}),
$$

where

$$
\text { MSE }=\frac{\sum_{i} \sum_{j}|X(i, j)-Y(i, j)|^{2}}{N}
$$

and where $X$ and $Y$ are input image and output image, respectively, $N$ is the total number of image pixels, and $L$ is the dynamic range of pixel values. The unit of PSNR is decibels $(\mathrm{dB})$.

The mean value is used to evaluate the average luminance of image. The higher mean value represents that the image is brighter. SD is also a popular metric in image enhancement and is used to estimate the contrast of image. The image contrast is grater if the SD value is higher. For mean and SD, we compute the average value of three channels of the color pathological image in our experiments.

EME is one well-known blind-reference image quality assessment (IQA) metric. It gives a quality score to each image based on the image contrast. The larger EME value represents the more detail information and more obvious variation in local region. The definition of EME is as follows:

$$
\mathrm{EME}_{k_{1}, k_{2}}=\frac{1}{k_{1}, k_{2}} \sum_{l=1}^{k_{2}} \sum_{k=1}^{k_{1}} 20 \log \frac{I_{\mathrm{max} ; k, l}^{\omega}}{I_{\mathrm{min} ; k, l}^{\omega}},
$$

where the test image is divided into $k_{1} \times k_{2}$ small blocks and $I_{\mathrm{max} ; k, l}^{\omega}$ and $I_{\mathrm{min} ; k, l}^{\omega}$ represent the maximum and minimum values of pixel, respectively, in block $\omega_{k, l}$.
Information entropy is an important metric to measure the content of image. And the higher value indicates an image with richer details. The equation of entropy is as follows:

$$
H=-\sum_{k=1}^{M} p_{k} \log _{2} p_{k}
$$

where $M$ is the gray levels of image and $p_{k}$ is the probability of gray level $k$ in the whole image.

All the objective evaluation result data is shown in Table 3 and all the data is an average value of our 140 pathological images. We can see from Table 3 that all these algorithms could enhance image to some extent. The SD values of $\mathrm{HE}-$ based methods are much higher than other methods. But their contribution is not outstanding when taking the serious distortion problem of those enhanced images into account. In addition, the PSNR value of our algorithm is not as good as the Retinex-based algorithms, but we obtain the best results in all the other metrics of mean, EME, and entropy. And considering that we just use the original image and the enhanced image to replace the noise-free image and test image in the definition of PSNR, the contribution of PSNR is limited. So in conclusion, our method is superior to others.

Moreover, in order to indicate the complexity of our algorithm, we compare the run times of all the algorithms, running in the same platform and using the same image as described above. The final average result is shown in the last column of Table 3. It is obvious that our algorithm is much faster than Retinex-based algorithms. Although the run time of HE-based algorithm is the least, the time cost of our algorithm is worthy in view of the quality of enhanced images.

4.3. Cell Segmentation. We also use a simple automatic cell segmentation method based on morphology to verify that our image enhancement algorithm can improve subsequent image segmentation and other image analysis processes. In this cell segmentation method, firstly transform the original RGB image into gray-scale image and then conduct image opening operation, image reconstruction, image binarization, image erosion and dilation operations, and image denoising [36]. We select 20 groups of enhanced 


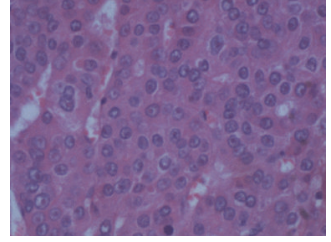

(a) Original image

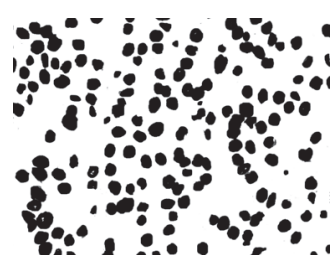

(b) Ground truth

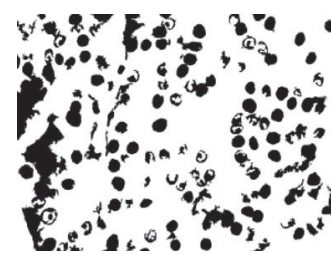

(c) Frankle-McCann

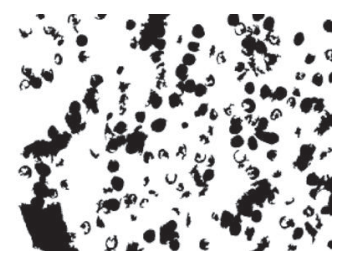

(d) SSR

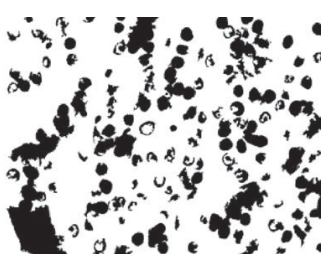

(e) MSR

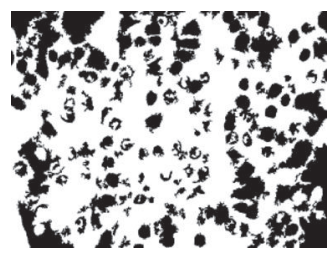

(f) DSIHE

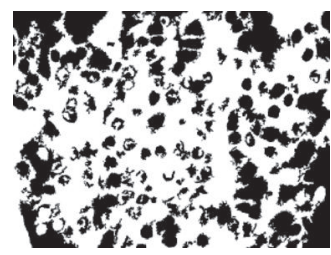

(g) MMBEBHE

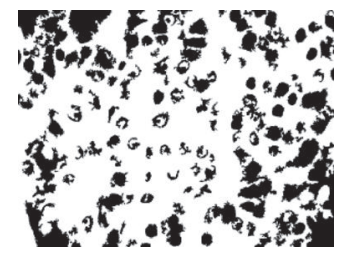

(h) RMSHE

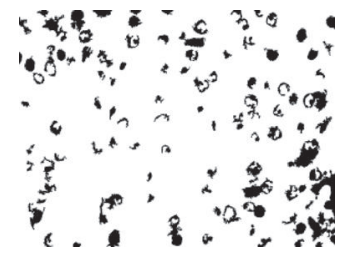

(i) RSIHE

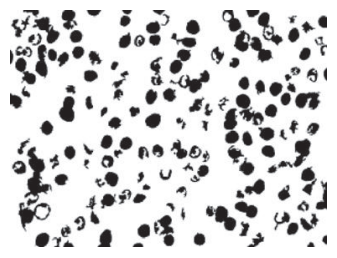

(j) Proposed

FIGURE 6: The cell segmentation results of enhanced images of different image enhancement algorithms.

TABLE 4: The average performance of segmentation results of different image enhancement algorithms.

\begin{tabular}{lcccccccc}
\hline \multirow{2}{*}{ Evaluations } & \multicolumn{9}{c}{ Algorithms } \\
& Frankle-McCann & SSR & MSR & DSIHE & MMBEBHE & RMSHE & RSIHE & Proposed \\
\hline Jaccard index & 0.8079 & 0.8079 & 0.7752 & 0.7132 & 0.6848 & 0.7318 & 0.7539 & $\mathbf{0 . 8 5 5 2}$ \\
Dice coefficient & 0.8937 & 0.8748 & 0.8734 & 0.8326 & 0.8129 & 0.8451 & 0.8597 & $\mathbf{0 . 9 9 2 0}$ \\
\hline
\end{tabular}

images in which the cell regions are relatively obvious to do test. Figure 6 demonstrates one group segmentation result, where Figure 6(a) is the original RGB pathological image, Figure $6(\mathrm{~b})$ is the ground truth segmented manually under the guidance of pathologists, and Figure 6(c)-Figure 6(j) are obtained from segmenting the corresponding enhanced images.

It is clear that the cell segmentation result of our algorithm is more close to the ground truth. In order to compare the segmentation results quantitatively, we adopt two standard segmentation metrics to evaluate, namely, dice coefficient [37] and Jaccard index [38]. The definitions of them are as follows:

$$
\begin{aligned}
\mathrm{DC}(\mathrm{SR}, \mathrm{TR}) & =\frac{2 \times \operatorname{Num}_{\left(\operatorname{pixel}_{\mathrm{SR}} \cap \mathrm{pixel}_{\mathrm{TR}}\right)}}{\operatorname{Num}\left(\operatorname{pixel}_{\mathrm{SR}}\right)+\operatorname{Num}_{\left(\mathrm{pixel}_{\mathrm{TR}}\right)},} \\
\mathrm{JI}(\mathrm{SR}, \mathrm{TR}) & =\frac{\operatorname{Num}\left(\operatorname{pixel}_{\mathrm{SR}} \cap \operatorname{pixel}_{\mathrm{TR}}\right)}{\operatorname{Num}\left(\operatorname{pixel}_{\mathrm{SR}} \cup \operatorname{pixel}_{\mathrm{TR}}\right)},
\end{aligned}
$$

where SR is the segmentation region, TR is the true region of the target, and Num(pixel) is the number of relevant pixel points. The average results of performance metrics are displayed in Table 4 . We can see that the result of our proposed method is the best. Our HDR pathological image enhancement method is superior to the comparison algorithms as we can improve the image segmentation and pathological analysis better.

In sum, we proposed that HDR pathological image enhancement method obtains a better result according to both pathologists' subjective evaluation and quantitative analysis in data, and the cell segmentation method also proves the better performance of our method, as the quality and detail of original image are both improved.

\section{Conclusions}

This paper proposes new HDR pathological image enhancement methods based on GIF and improved bias field correction model. First, stain normalization and wavelet denoising operations are used in image preprocessing. And the improved bias field model is introduced to correct the intensity inhomogeneity and detail discontinuity of image. Then the HDR pathological image is generated using LDR image and $\mathrm{H}$ and $\mathrm{E}$ channel images. Next, the $Y$ component of HDR image is separated into base layer and detail layer by GIF and the two layers are enhanced separately. Finally, the fine enhanced image is acquired after combining the $Y$ component and the color components. To verify the effectiveness of the proposed method, we perform the enhancement experiments using 140 pathological images. The experiment results and comparisons with related work demonstrate that our proposed method improves the image quality in terms of human vision, PSNR, SD, mean, EME, information entropy, and cell segmentation.

\section{Competing Interests}

The authors declare that there were no competing interests regarding the publication of this article. 


\section{Acknowledgments}

This work was supported by the National Natural Science Foundation of China (no. 61472073).

\section{References}

[1] S. M. Pizer, E. P. Amburn, J. D. Austin et al., "Adaptive histogram equalization and its variations," Computer Vision, Graphics, \& Image Processing, vol. 39, no. 3, pp. 355-368, 1987.

[2] V. Vani and K. V. M. Prashanth, "Color image enhancement techniques in Wireless Capsule Endoscopy," in Proceedings of the IEEE International Conference on Trends in Automation, Communications and Computing Technology (I-TACT '15), vol. 1, pp. 1-6, Bangalore, India, December 2015.

[3] H. Cao, L. Tian, J. Liu, H. Wang, and S. Feng, "Color image enhancement using power-constraint histogram equalization for AMOLED," in Proceedings of the IEEE 11th International Conference on ASIC (ASICON '15), pp. 1-4, IEEE, Chengdu, China, November 2015.

[4] N. M. Kwok, G. Fang, and H. Y. Shi, "Color enhancement for images from digital camera using a transformation-free approach," in Proceedings of the 9th International Conference on Sensing Technology (ICST '15), pp. 168-172, IEEE, Auckland, New Zealand, December 2015.

[5] S. D. Nikam and R. U. Yawale, "Color image enhancement using daubechies wavelet transform and HIS color model," in Proceedings of the International Conference on Industrial Instrumentation and Control (ICIC '15), pp. 1323-1327, IEEE, Pune, India, May 2015.

[6] L. G. Villanueva, G. M. Callicó, F. Tobajas et al., "Medical diagnosis improvement through image quality enhancement based on super-resolution," in Proceedings of the 13th Euromicro Conference on Digital System Design: Architectures, Methods and Tools (DSD '10), pp. 259-262, IEEE, Lille, France, September 2010.

[7] W. Sun, F. Li, and Q. Zhang, "The applications of improved retinex algorithm for X-ray medical image enhancement," in Proceedings of the International Conference on Computer Science and Service System (CSSS '12), pp. 1655-1658, IEEE, Nanjing, China, August 2012.

[8] G. Zhang, D. Sun, P. Yan, H. Zhao, and Z. Li, "A LDCT image contrast enhancement algorithm based on single-scale retinex theory," in Proceedings of the International Conference on Computational Intelligence for Modelling Control \& Automation, pp. 1282-1287, IEEE Computer Society, Vienna, Austria, December 2008.

[9] S. Setty, N. K. Srinath, and M. C. Hanumantharaju, "Development of multiscale retinex algorithm for medical image enhancement based on multi-rate sampling," in Proceedings of the International Conference on Signal Processing Image Processing \& Pattern Recognition, pp. 145-150, 2013.

[10] J. Mccann, "Lessons learned from mondrians applied to real images and color gamuts," in Proceedings of the Color and Imaging Conference, vol. 8, pp. 1-8, 1999.

[11] B. V. Funt, F. Ciurea, and J. J. McCann, "Retinex in Matlab," in Proceedings of the Color and Imaging Conference, pp. 112-121, Scottsdale, Ariz, USA, November 2000.

[12] K. Kim, J. Bae, and J. Kim, "Natural hdr image tone mapping based on retinex," IEEE Transactions on Consumer Electronics, vol. 57, no. 4, pp. 1807-1814, 2011.
[13] J. Kuang, G. M. Johnson, and M. D. Fairchild, "iCAM06: a refined image appearance model for HDR image rendering," Journal of Visual Communication \& Image Representation, vol. 18, no. 5, pp. 406-414, 2007.

[14] M.-L. Song, H.-Q. Wang, C. Chen, X.-Q. Ye, and W.-K. Gu, "Tone mapping for high dynamic range image using a probabilistic model," Journal of Software, vol. 20, no. 3, pp. 734-743, 2010.

[15] F. Branchitta, M. Diani, G. Corsini, and M. Romagnoli, "New technique for the visualization of high dynamic range infrared images," Optical Engineering, vol. 48, no. 9, Article ID 096401, 2009.

[16] C. Zuo, "Display and detail enhancement for high-dynamicrange infrared images," Optical Engineering, vol. 50, no. 12, Article ID 127401, pp. 895-900, 2011.

[17] K. He, J. Sun, and X. Tang, "Guided image filtering," IEEE Transactions on Pattern Analysis and Machine Intelligence, vol. 35, no. 6, pp. 1397-1409, 2013.

[18] N. Liu and D. Zhao, "Detail enhancement for high-dynamicrange infrared images based on guided image filter," Infrared Physics \& Technology, vol. 67, pp. 138-147, 2014.

[19] C. Li, R. Huang, Z. Ding et al., "A level set method for image segmentation in the presence of intensity inhomogeneities with application to MRI," IEEE Transactions on Image Processing, vol. 20, no. 7, pp. 2007-2016, 2011.

[20] C. Li, J. C. Gore, and C. Davatzikos, "Multiplicative intrinsic component optimization (MICO) for MRI bias field estimation and tissue segmentation," Magnetic Resonance Imaging, vol. 32, no. 7, pp. 913-923, 2014.

[21] A. Vahadane, T. Peng, A. Sethi et al., "Structure-preserving color normalization and sparse stain separation for histological images," IEEE Transactions on Medical Imaging, vol. 35, no. 8, pp. 1962-1971, 2016.

[22] E. Reinhard, M. Ashikhmin, B. Gooch, and P. Shirley, "Color transfer between images," IEEE Computer Graphics \& Applications, vol. 21, no. 5, pp. 34-41, 2001.

[23] D. L. Donoho, "De-noising by soft-thresholding," IEEE Transactions on Information Theory, vol. 41, no. 3, pp. 613-627, 1995.

[24] E. Zhang, H. Yang, and M. Xu, "A novel tone mapping method for high dynamic range image by incorporating edgepreserving filter into method based on retinex," Applied Mathematics \& Information Sciences, vol. 9, no. 1, pp. 411-417, 2015.

[25] M. Macenko, M. Niethammer, J. S. Marron et al., "A method for normalizing histology slides for quantitative analysis," in Proceedings of the IEEE International Conference on Symposium on Biomedical Imaging: From Nano To Macro, pp. 1107-1110, IEEE Press, 2009.

[26] T. Mitsunaga and S. K. Nayar, "Radiometric self calibration," in Proceedings of the IEEE Computer Society Conference on Computer Vision and Pattern Recognition, vol. 1, p. 1374, Fort Collins, Colo, USA, June 1999.

[27] F. Durand and J. Dorsey, "Fast bilateral filtering for the display of high-dynamic-range images," ACM Transactions on Graphics, vol. 21, no. 3, pp. 257-266, 2002.

[28] D. J. Jobson, Z.-U. Rahman, and G. A. Woodell, "Properties and performance of a center/surround retinex," IEEE Transactions on Image Processing, vol. 6, no. 3, pp. 451-462, 1997.

[29] D. J. Jobson, Z.-U. Rahman, and G. A. Woodell, "A multiscale retinex for bridging the gap between color images and the human observation of scenes," IEEE Transactions on Image Processing, vol. 6, no. 7, pp. 965-976, 1997. 
[30] Y. Wang, Q. Chen, and B. Zhang, "Image enhancement based on equal area dualistic sub-image histogram equalization method," IEEE Transactions on Consumer Electronics, vol. 45, no. 1, pp. 68-75, 1999.

[31] S.-D. Chen and A. R. Ramli, "Minimum mean brightness error bi-histogram equalization in contrast enhancement," IEEE Transactions on Consumer Electronics, vol. 49, no. 4, pp. 13101319, 2003.

[32] S.-D. Chen and A. R. Ramli, "Contrast enhancement using recursive mean-separate histogram equalization for scalable brightness preservation," IEEE Transactions on Consumer Electronics, vol. 49, no. 4, pp. 1301-1309, 2003.

[33] K. S. Sim, C. P. Tso, and Y. Y. Tan, "Recursive sub-image histogram equalization applied to gray scale images," Pattern Recognition Letters, vol. 28, no. 10, pp. 1209-1221, 2007.

[34] V. L. Jaya and R. Gopikakumari, "IEM: a new image enhancement metric for contrast and sharpness measurements," International Journal of Computer Applications, vol. 79, no. 9, pp. 1-9, 2013.

[35] S. S. Agaian, K. Panetta, and A. M. Grigoryan, "Transformbased image enhancement algorithms with performance measure," IEEE Transactions on Image Processing, vol. 10, no. 3, pp. 367-382, 2001.

[36] M. Braiki, A. Benzinou, K. Nasreddine, S. Labidi, and N. Hymery, "Segmentation of dendritic cells from microscopic images using mathematical morphology," in Proceedings of the 2nd International Conference on Advanced Technologies for Signal and Image Processing (ATSIP '16), pp. 282-287, Monastir, Tunisia, March 2016.

[37] L. R. Dice, "Measures of the amount of ecologic association between species," Ecology, vol. 26, no. 3, pp. 297-302, 1945.

[38] P. Jaccard, "The distribution of the flora in the alpine zone," New Phytologist, vol. 11, no. 2, pp. 37-50, 1912. 


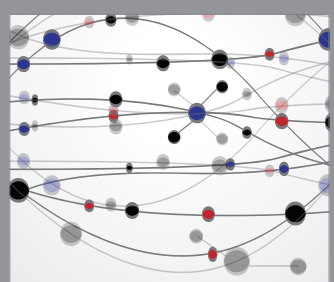

The Scientific World Journal
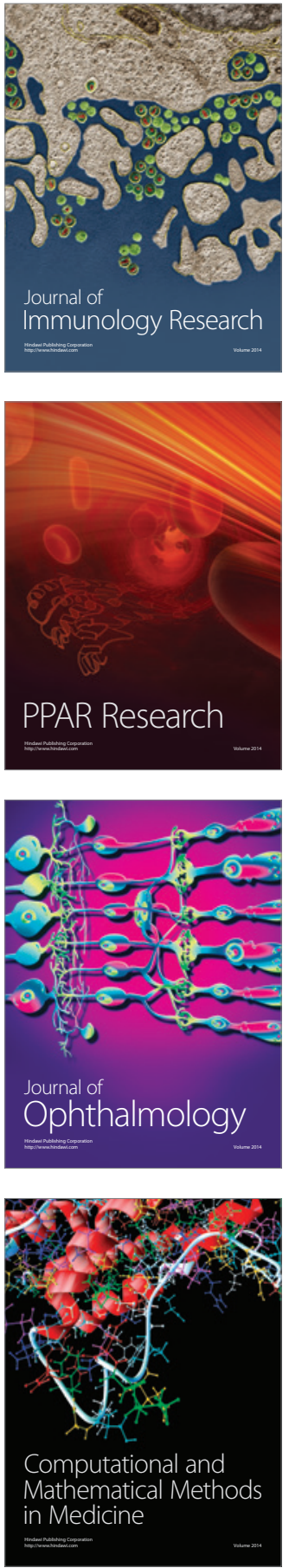

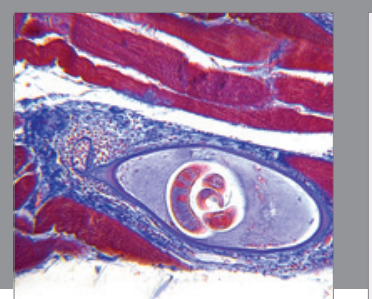

Gastroenterology Research and Practice

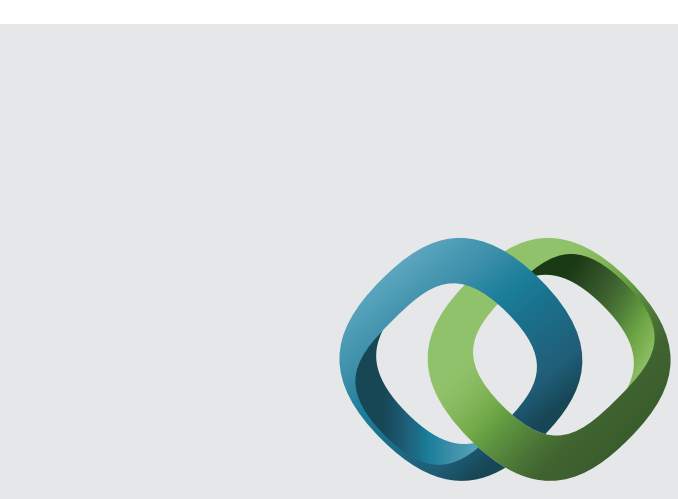

\section{Hindawi}

Submit your manuscripts at

http://www.hindawi.com
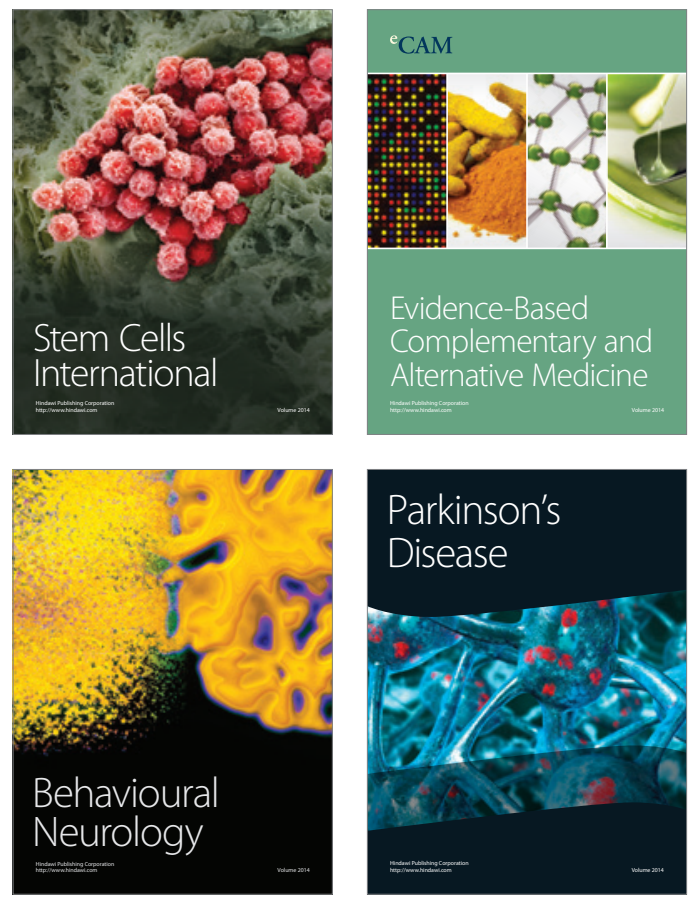
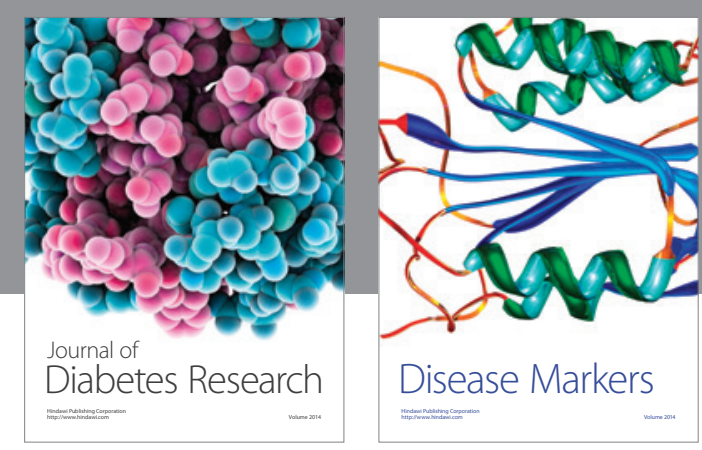

Disease Markers
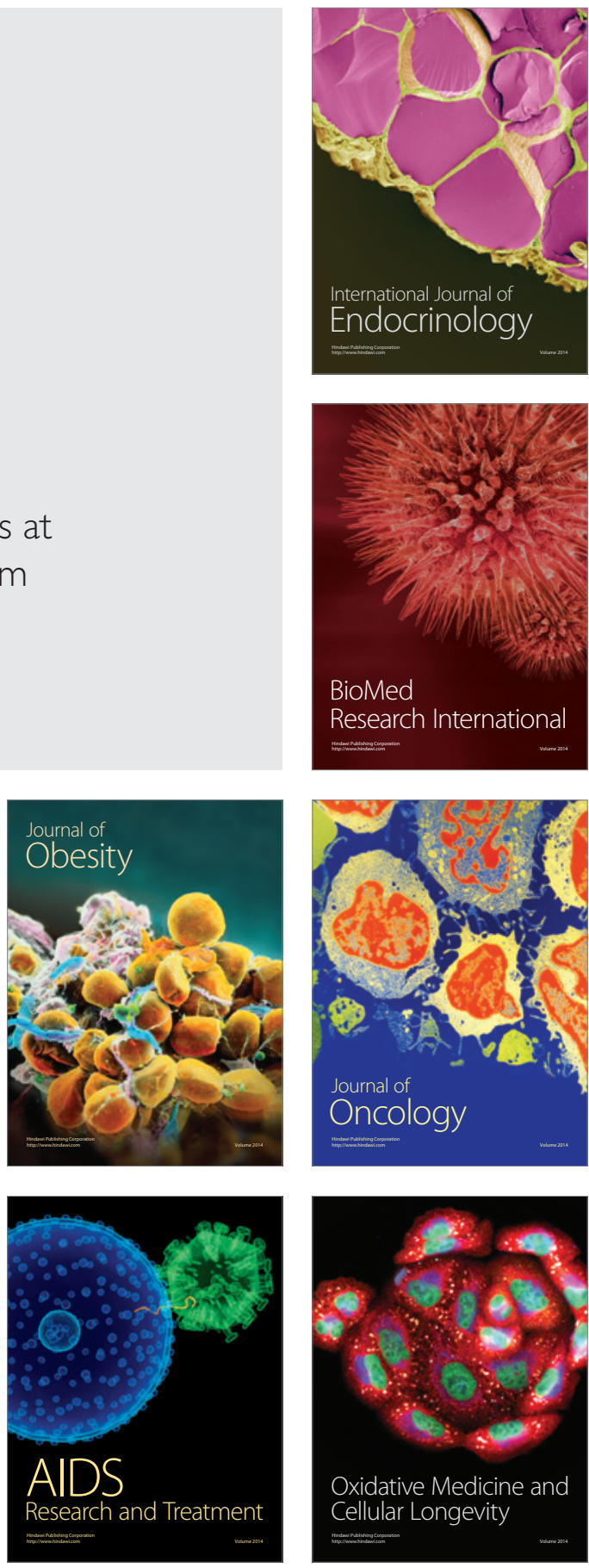